\title{
Genetic polymorphisms in the telomere length-related gene ACYP2 are associated with the risk of colorectal cancer in a Chinese Han population
}

\author{
Fang Liu ${ }^{1,2, *}$, Zhongguo Zhang ${ }^{1,2, *}$, Yong Zhang ${ }^{3}$, Yue Chen², Xiaoyu Yang ${ }^{2}$, Jibin Li², \\ Jiaxing Zhao ${ }^{1}$ \\ ${ }^{1}$ Large-Scale Data Analysis Center of Cancer Precision Medicine, Cancer Hospital of China Medical University, Liaoning Cancer \\ Hospital \& Institute, Dadong District, Shenyang 110042, Liaoning Province, P R China \\ ${ }^{2}$ Department of Colorectal Cancer Surgery, Cancer Hospital of China Medical University, Liaoning Cancer Hospital \& Institute, \\ Dadong District, Shenyang 110042, Liaoning Province, P R China \\ ${ }^{3}$ Department of Pathology, Cancer Hospital of China Medical University, Liaoning Cancer Hospital \& Institute, Dadong District, \\ Shenyang 110042, Liaoning Province, P R China \\ *These authors have contributed equally to this work \\ Correspondence to: Zhongguo Zhang, email: zhangzhongguoln@163.com \\ Keywords: single nucleotide polymorphism (SNP), ACYP2, telomere length, colorectal cancer, association study \\ Received: October 09, $2016 \quad$ Accepted: November 24, 2016 \\ Published: December 25, 2016
}

\section{ABSTRACT}

We investigated the association between single nucleotide polymorphisms (SNPs) in ACYP2, which has been associated with telomere length in several types of cancer, and the risk of CRC in a Chinese Han population. In a case-control study that included 247 cases and 300 healthy controls, 14 SNPs in ACYP2 were selected and genotyped using the Sequenom MassARRAY platform. Odds ratios (ORs) and $95 \%$ confidence intervals (CIs) were calculated using unconditional logistic regression after adjusting for age and gender. We determined that rs843711 and rs843706 were associated with an increased risk of CRC ( $r s 843711$ : OR $=1.376,95 \%$ CI $=1.082-1.749, p=0.009$; rs843706: $\mathrm{OR}=1.361,95 \% \mathrm{CI}=1.069-1.733, p=0.012)$. Additionally, rs6713088, rs843645, rs843711, and rs843706 were associated with an increased risk of CRC under additive and recessive models $(p<0.05)$. Finally, the "TTCTCGCC" and "CG" haplotypes decreased the risk of CRC, while the "AG" haplotype increase the risk of CRC. The association between rs843711 and CRC remained significant after Bonferroni correction for multiple comparisons ( $p \leq 0.00036)$. Our data shed new light on the associations between genetic variants in the ACYP2 gene and CRC susceptibility in a Chinese Han population.

\section{INTRODUCTION}

Colorectal cancer (CRC) is the third most common cancer and the fourth leading cause of cancer deaths worldwide. The incidence of CRC is increasing each year [1]. Although the development of novel therapeutics has increased the 5-year survival rate to $90 \%$ for patients who are diagnosed at stage I, the prognosis of patients with advanced stage CRC is poor [2]. Therefore, the identification of epidemiological factors that influence the development of CRC will enable facilitate prevention and early detection, resulting in improve patient outcomes. Twin studies have shown that the contribution of inherited factors (mainly genetic) to the etiology of $\mathrm{CRC}$ is approximately $35 \%$ [3], which means that genetic factors significantly impact CRC susceptibility.

Telomeres are nucleoprotein complexes at the ends of eukaryotic chromosomes. These structures consist of noncoding TTAGGG repeats and associated telomere binding proteins. Telomeres maintain chromosome integrity and genomic stability by preventing nucleolytic degradation, chromosomal end-to-end fusion, and irregular recombination [4-6]. Telomere length is determined by a balance between processes that shorten and lengthen the telomere [7]. The average telomere length ranges from $10-15 \mathrm{~kb}$ in human somatic cells. Telomere length is 
reduced by 50-200 bp with each cell division [8]. During somatic cell replication, telomere length progressively shortens due to the inability of DNA polymerase to fully replicate the 3' end of the DNA. In general, a critically short telomere length can trigger replicative senescence and cell death [9]. This can result in genomic instability and chromosomal abnormalities, which can promote carcinogenesis [10].

The ACYP2 gene, located on chromosome 2p16.2, encodes a small cytosolic acylphosphatase enzyme that catalyzes the hydrolysis of carboxyl-phosphate bonds [11]. Genome wide association studies have demonstrated that genetic polymorphisms in $A C Y P 2$ are associated with telomere length [12], which has led to studies of the association between $A C Y P 2$ and various cancers. For example, rs11125529 in $A C Y P 2$ was found to be associated with the risk of several hormone-related cancers (e.g. breast, ovarian, and prostate) in a European population $[13,14]$. However, few studies have investigated the association between genetic variants in $A C Y P 2$ and the risk of CRC. We performed a case-control study to analyze the association between 14 single nucleotide polymorphisms (SNPs) in $A C Y P 2$ and the risk of $\mathrm{CRC}$ in a Chinese Han population.

\section{RESULTS}

A total of 247 CRC cases (107 men and 140 women; mean age, $58.32 \pm 12.75$ years) and 300 controls (180 men and 120 women; mean age, $60.42 \pm 5.14$ years) were included in the study. The clinical characteristics of the cases and controls are shown in Table 1. There were no significant differences in the age and gender distributions between the case and control groups $(p<0.05)$. The minor allele frequencies (MAFs) of the analyzed SNPs in the case and control groups are shown in Table 2. All SNPs were in Hardy-Weinberg equilibrium (HWE) in the controls $(p>0.05)$ with the exception of rs843740, which was excluded from subsequent analyses. The MAFs of the SNPs in the control group were similar to those reported for the HapMap Asian population. Using chi-square tests, we determined that rs843711 was associated with a 1.376fold increase in the risk of CRC (95\% confidence interval $[\mathrm{CI}]=1.082-1.749 ; p=0.009)$. Similarly, rs843706 was associated with a significant increase in the risk of CRC (odds ratio $[\mathrm{OR}]=1.361,95 \% \mathrm{CI}=1.069-1.733 ; p=$ 0.012 ). No significant associations were detected between the other SNPs and CRC risk.

The genotype frequencies of the $A C Y P 2$ polymorphisms are shown in Table 3. Compared to the CC genotype, the frequency of the GG genotype of rs6713088 polymorphism in the case group significantly differed from the controls (GG vs. CC: $\mathrm{OR}=1.750,95 \% \mathrm{CI}=1.032$ 2.967; $p=0.038$ ), suggesting that rs6713088 increased the risk of CRC. Similarly, compared to individuals with the CC genotype of rs843711, individuals with the TT genotype had a significantly increased risk of CRC (TT vs. $\mathrm{CC}: \mathrm{OR}=2.007,95 \% \mathrm{CI}=1.218-3.308 ; p=0.006)$. Individuals with the AA genotype of rs843706 also had an increased risk of CRC compared to those with the CC genotype (AA vs. CC: $\mathrm{OR}=1.971,95 \% \mathrm{CI}=1.184-3.280$; $p=0.009$ ).

We assumed that the minor allele of each SNP was a risk factor compared to the wild-type allele. Three genetic models (dominant, recessive, and additive) were applied to analyze the associations between the SNPs and CRC risk using an unconditional logistic regression analysis with adjustments for age and gender (Table 4). We found that the minor allele $(\mathrm{G})$ of rs6713088 and rs843645 was associated with an increased risk of CRC under the additive model ( $\mathrm{rs} 6713088$ : $\mathrm{OR}=1.304,95 \% \mathrm{CI}=$ $1.012-1.681 ; p=0.04 . \mathrm{rs} 843645: \mathrm{OR}=1.322,95 \% \mathrm{CI}=$ $1.001-1.746 ; p=0.049)$. The minor allele $(\mathrm{T})$ of $\mathrm{rs} 843711$ was associated with an increased risk of CRC under the additive model $(\mathrm{OR}=1.38,95 \% \mathrm{CI}=1.077-1.768 ; p=$ $0.011)$ and recessive model $(\mathrm{OR}=1.921,95 \% \mathrm{CI}=1.247$ $2.960 ; p=0.003$ ). Additionally, the minor allele (A) of rs843706 was associated with a significantly increased risk of $\mathrm{CRC}$ under the additive model $(\mathrm{OR}=1.39,95 \%$ $\mathrm{CI}=1.079-1.791 ; p=0.011)$ and recessive model $(\mathrm{OR}=$ $1.703,95 \% \mathrm{CI}=1.101-2.633 ; p=0.017$ ). However, only rs843711 remained significant after Bonferroni correction for multiple comparisons ( $p \leq 0.00036)$.

We further characterized the SNPs in $A C Y P 2$ SNPs using linkage disequilibrium (LD) and haplotype analyses. Pairwise LD was calculated between all 13 SNPs and the haplotype structure of the $A C Y P 2$ gene was analyzed (D' and $\mathrm{r}^{2}$ ). Haplotype blocks were divided using the D' confidence interval method. Adjacent SNPs with D' values and 95\% CIs between 0.70-0.98 were classified as the same haplotype block. Two LD blocks were detected in the control group (Figure 1). Block 1 consisted of 8 closely linked SNPs (rs1682111, rs843752, rs10439478, rs843645, rs11125529, rs12615793, rs843711, and rs11896604) and block 2 consisted of two linked SNPs (rs843706 and rs17045754).

Finally, a haplotype-based association study was performed to evaluate the relationship between $A C Y P 2$ haplotypes and risk of CRC (Table 5). The "TTCTCGCC" haplotype was associated with a decreased risk of CRC $(\mathrm{OR}=0.719 ; 95 \% \mathrm{CI}, 0.529-0.978, p=0.035)$. This haplotype consisted of rs1682111, rs843752, rs10439478, rs843645, rs11125529, rs12615793, rs843711, and rs11896604. Additionally, the "AG" haplotype of rs843706 and rs17045754 was associated with an increased risk of $\mathrm{CRC}(\mathrm{OR}=1.377 ; 95 \% \mathrm{CI}, 1.044-1.815, p=0.023)$. In contrast, the "CG" haplotype was associated with a decreased risk of CRC $(\mathrm{OR}=0.732 ; 95 \% \mathrm{CI}, 0.568-0.944$, $p=0.016)$. 
Table 1: Characteristics of the cases and controls included in the study

\begin{tabular}{|c|c|c|c|c|}
\hline Variables & Case $(N=247)$ & Control $(\mathrm{N}=\mathbf{3 0 0})$ & Total & $p$ value \\
\hline Sex, No. $(\%)$ & & & & $<0.001^{\mathrm{a}}$ \\
\hline Male & $107(43.3)$ & $180(60)$ & $287(52.5)$ & \\
\hline Female & $140(56.7)$ & $120(40)$ & $260(47.5)$ & \\
\hline Mean age \pm SD & $58.32 \pm 12.75$ & $60.42 \pm 5.14$ & & $0.015^{\mathrm{b}}$ \\
\hline
\end{tabular}

a The $p$ value was calculated using Pearson's chi-square tests.

$\mathrm{b}$ The $p$ value was calculated using Welch's t tests.

$\mathrm{SD}$, standard deviation.

Table 2: Allele frequencies in cases and controls and odds ratio estimates for colorectal cancer

\begin{tabular}{|c|c|c|c|c|c|c|c|c|c|}
\hline \multirow{2}{*}{ SNP ID } & \multirow{2}{*}{ Gene (s) } & \multirow{2}{*}{ Band } & \multirow{2}{*}{ Alleles A $\mathbf{A}^{\mathrm{a}} / \mathbf{B}$} & \multicolumn{2}{|c|}{ MAF } & \multirow{2}{*}{$\begin{array}{c}\text { HWE } \\
p \text {-value }\end{array}$} & \multirow{2}{*}{ ORs } & \multirow{2}{*}{$95 \% \mathrm{CI}$} & \multirow{2}{*}{$p$-value } \\
\hline & & & & Case & Control & & & & \\
\hline rs6713088 & $A C Y P 2$ & $2 \mathrm{p} 16.2$ & $\mathrm{G} / \mathrm{C}$ & 0.429 & 0.377 & 0.623 & 1.243 & $0.974-1.585$ & 0.080 \\
\hline rs 12621038 & $A C Y P 2$ & $2 \mathrm{p} 16.2$ & $\mathrm{~T} / \mathrm{C}$ & 0.411 & 0.450 & 0.415 & 0.852 & $0.669-1.084$ & 0.193 \\
\hline rs1682111 & $A C Y P 2$ & $2 \mathrm{p} 16.2$ & $\mathrm{~A} / \mathrm{T}$ & 0.309 & 0.333 & 0.604 & 0.894 & $0.692-1.155$ & 0.391 \\
\hline rs843752 & $A C Y P 2$ & $2 \mathrm{p} 16.2$ & $\mathrm{G} / \mathrm{T}$ & 0.287 & 0.248 & 0.877 & 1.216 & $0.929-1.592$ & 0.154 \\
\hline rs 10439478 & $A C Y P 2$ & $2 \mathrm{p} 16.2$ & $\mathrm{C} / \mathrm{A}$ & 0.409 & 0.425 & 0.237 & 0.937 & $0.736-1.193$ & 0.597 \\
\hline rs843645 & $A C Y P 2$ & $2 \mathrm{p} 16.2$ & $\mathrm{G} / \mathrm{T}$ & 0.285 & 0.237 & 0.749 & 1.283 & $0.978-1.683$ & 0.072 \\
\hline rs11125529 & $A C Y P 2$ & $2 \mathrm{p} 16.2$ & $\mathrm{~A} / \mathrm{C}$ & 0.198 & 0.170 & 0.411 & 1.208 & $0.889-1.643$ & 0.227 \\
\hline rs12615793 & $A C Y P 2$ & $2 \mathrm{p} 16.2$ & $\mathrm{~A} / \mathrm{G}$ & 0.209 & 0.185 & 0.254 & 1.161 & $0.860-1.565$ & 0.329 \\
\hline rs843711 & $A C Y P 2$ & $2 \mathrm{p} 16.2$ & $\mathrm{~T} / \mathrm{C}$ & 0.494 & 0.415 & 0.287 & 1.376 & $1.082-1.749$ & $0.009 *$ \\
\hline rs11896604 & $A C Y P 2$ & $2 \mathrm{p} 16.2$ & $\mathrm{G} / \mathrm{C}$ & 0.213 & 0.177 & 1.000 & 1.258 & $0.931-1.699$ & 0.134 \\
\hline rs843706 & $A C Y P 2$ & $2 \mathrm{p} 16.2$ & $\mathrm{~A} / \mathrm{C}$ & 0.496 & 0.419 & 0.341 & 1.361 & $1.069-1.733$ & $0.012 *$ \\
\hline rs17045754 & $A C Y P 2$ & $2 \mathrm{p} 16.2$ & $\mathrm{C} / \mathrm{G}$ & 0.192 & 0.172 & 0.547 & 1.149 & $0.844-1.564$ & 0.378 \\
\hline rs843740 & $A C Y P 2$ & $2 \mathrm{p} 16.2$ & $\mathrm{~A} / \mathrm{G}$ & 0.453 & 0.403 & $<0.001^{\#}$ & 1.227 & $0.965-1.561$ & 0.095 \\
\hline rs843720 & $A C Y P 2$ & $2 \mathrm{p} 16.2$ & $\mathrm{G} / \mathrm{T}$ & 0.358 & 0.358 & 0.451 & 0.999 & $0.780-1.282$ & 0.999 \\
\hline
\end{tabular}

MAF, minor allelic frequency; HWE, Hardy-Weinberg Equilibrium; OR, odds ratio; CI: confidence interval.

a Minor allele; ${ }^{*}$ HWE $p$-value $\leq 0.05$ was excluded; ${ }^{*} p$ value $\leq 0.05$.

Bonferroni correction was performed with $p \leq 0.00036(0.05 / 14)$ considered significant.

\section{DISCUSSION}

We investigated the associations between 14 SNPs in the $A C Y P 2$ gene and the risk of CRC in a Chinese Han population. We determined that four SNPs (rs6713088, rs843645, rs843711, and rs843706) were associated with an increased risk of CRC. Additionally, we demonstrated that three haplotypes in $A C Y P 2$ were associated with CRC risk.

Previous studies have revealed an association between the $A C Y P 2$ gene and cancer cell metabolism (i.e. pyruvate metabolism and glycolysis/gluconeogenesis) $[15,16]$. Cancer cell survival and proliferation is associated with glucose uptake and altered expression of several metabolic intermediates in the glycolysis/ gluconeogenesis pathway such as phosphofructokinase (PFK) and pyruvate kinase $[17,18]$. PFK is essential for glycolysis and is a possible target for anticancer drugs. The transition of cancer cells to a highly proliferative state is correlated with reduced PFK expression [19]. Furthermore, pyruvate metabolism can generate lactate or alanine and is accompanied by the conversion of NADH to $\mathrm{NAD}^{+}$, which could provide substrates and energy for cancer cells to drive proliferation and differentiation [20]. Thus, stimulation of the pyruvate metabolic pathway may promote malignant transformation [18, 21]. We found that SNPs in ACPY2 were associated with CRC risk. Our data suggest that $A C P Y 2$ may play an important role 
Table 3: Genotype distributions of the SNPs and their associations with the risk of colorectal cancer

\begin{tabular}{|c|c|c|c|c|c|c|c|c|}
\hline \multirow{2}{*}{ SNP ID } & \multirow{2}{*}{$\begin{array}{c}\text { Alleles } \\
\mathbf{A}^{\mathrm{a} / \mathbf{B}}\end{array}$} & \multirow{2}{*}{ Genotype } & \multicolumn{2}{|c|}{ No. (frequency) } & \multicolumn{2}{|c|}{ Without adjustment } & \multicolumn{2}{|c|}{ With adjustment } \\
\hline & & & Case (\%) & Control (\%) & OR $(95 \%$ CI $)$ & $P$ & OR $(95 \% \mathrm{CI})$ & $P^{b}$ \\
\hline \multirow[t]{3}{*}{ rs6713088 } & $\mathrm{G} / \mathrm{C}$ & $\mathrm{CC}$ & $80(32.5)$ & $114(38)$ & 1 & & 1 & \\
\hline & & GG & $45(18.3)$ & $40(13.3)$ & $1.603(0.960-2.678)$ & 0.071 & $1.750(1.032-2.967)$ & $0.038^{*}$ \\
\hline & & $\mathrm{GC}$ & $121(49.2)$ & $146(48.7)$ & $1.181(0.813-1.716)$ & 0.383 & $1.232(0.840-1.807)$ & 0.286 \\
\hline \multirow[t]{3}{*}{ rs 12621038} & $\mathrm{~T} / \mathrm{C}$ & $\mathrm{CC}$ & $86(34.9)$ & $94(31.4)$ & 1 & & 1 & \\
\hline & & $\mathrm{TT}$ & $42(17.1)$ & $64(21.4)$ & $0.717(0.441-1.167)$ & 0.181 & $0.686(0.417-1.129)$ & 0.138 \\
\hline & & $\mathrm{TC}$ & $118(48)$ & $141(47.2)$ & $0.915(0.625-1.339)$ & 0.647 & $0.859(0.581-1.271)$ & 0.447 \\
\hline \multirow[t]{3}{*}{ rs1682111 } & $\mathrm{A} / \mathrm{T}$ & $\mathrm{TT}$ & $117(47.6)$ & $131(43.7)$ & 1 & & 1 & \\
\hline & & AA & $23(9.3)$ & $31(10.3)$ & $0.831(0.459-1.505)$ & 0.541 & $0.877(0.478-1.608)$ & 0.671 \\
\hline & & AT & $106(43.1)$ & $138(46)$ & $0.860(0.603-1.227)$ & 0.406 & $0.894(0.621-1.286)$ & 0.545 \\
\hline \multirow[t]{3}{*}{ rs843752 } & $\mathrm{G} / \mathrm{T}$ & TT & $124(50.4)$ & $170(56.7)$ & 1 & & 1 & \\
\hline & & GG & $19(7.7)$ & $19(6.3)$ & $1.371(0.697-2.697)$ & 0.301 & $1.385(0.692-2.77)$ & 0.358 \\
\hline & & GT & $103(41.9)$ & $111(37)$ & $1.272(0.893-1.813)$ & 0.183 & $1.322(0.920-1.901)$ & 0.132 \\
\hline \multirow[t]{3}{*}{ rs10439478 } & $\mathrm{C} / \mathrm{A}$ & AA & $83(33.6)$ & $104(34.8)$ & 1 & & 1 & \\
\hline & & $\mathrm{CC}$ & $38(15.4)$ & $59(19.7)$ & $0.807(0.490-1.330)$ & 0.401 & $0.712(0.427-1.190)$ & 0.195 \\
\hline & & $\mathrm{CA}$ & $126(51)$ & $136(45.5)$ & $1.161(0.797-1.692)$ & 0.438 & $1.118(0.760-1.645)$ & 0.570 \\
\hline \multirow[t]{3}{*}{ rs843645 } & $\mathrm{G} / \mathrm{T}$ & $\mathrm{TT}$ & $126(51.2)$ & $176(58.7)$ & 1 & & 1 & \\
\hline & & GG & $20(8.1)$ & $18(6)$ & $1.552(0.789-3.053)$ & 0.203 & $1.622(0.811-3.246)$ & 0.172 \\
\hline & & GT & $100(40.7)$ & $106(35.3)$ & $1.318(0.923-1.882)$ & 0.129 & $1.380(0.958-1.988)$ & 0.084 \\
\hline \multirow[t]{3}{*}{ rs11125529 } & $\mathrm{A} / \mathrm{C}$ & $\mathrm{CC}$ & $160(64.8)$ & $204(68)$ & 1 & & 1 & \\
\hline & & AA & $11(4.5)$ & $6(2)$ & $2.338(0.846-6.457)$ & 0.101 & $2.631(0.933-7.417)$ & 0.067 \\
\hline & & $\mathrm{AC}$ & $76(30.7)$ & $90(30)$ & $1.077(0.745-1.557)$ & 0.695 & $1.003(0.686-1.465)$ & 0.988 \\
\hline \multirow[t]{3}{*}{ rs12615793 } & $\mathrm{A} / \mathrm{G}$ & GG & $156(63.1)$ & $196(65.3)$ & 1 & & 1 & \\
\hline & & AA & $12(4.9)$ & $7(2.4)$ & $2.154(0.828-5.600)$ & 0.116 & $2.384(0.899-6.314)$ & 0.081 \\
\hline & & $\mathrm{AG}$ & $79(32)$ & $97(32.3)$ & $1.023(0.711-1.472)$ & 0.901 & $0.953(0.655-1.385)$ & 0.799 \\
\hline \multirow[t]{3}{*}{ rs843711 } & $\mathrm{T} / \mathrm{C}$ & $\mathrm{CC}$ & $67(27.2)$ & $98(32.7)$ & 1 & & 1 & \\
\hline & & $\mathrm{TT}$ & $64(26)$ & $47(15.7)$ & $1.992(1.222-3.245)$ & $0.006^{*}$ & $2.007(1.218-3.308)$ & $0.006^{*}$ \\
\hline & & $\mathrm{TC}$ & $115(46.8)$ & $155(51.6)$ & $1.085(0.732-1.608)$ & 0.684 & $1.073(0.718-1.603)$ & 0.732 \\
\hline \multirow[t]{3}{*}{ rs11896604 } & $\mathrm{G} / \mathrm{C}$ & $\mathrm{CC}$ & $156(63.2)$ & $203(67.7)$ & 1 & & 1 & \\
\hline & & GG & $14(5.7)$ & $9(3)$ & $2.024(0.854-4.798)$ & 0.109 & $2.219(0.915-5.384)$ & 0.078 \\
\hline & & $\mathrm{GC}$ & $77(31.1)$ & $88(29.3)$ & $1.139(0.786-1.649)$ & 0.492 & $1.050(0.718-1.537)$ & 0.800 \\
\hline \multirow[t]{3}{*}{ rs843706 } & $\mathrm{A} / \mathrm{C}$ & $\mathrm{CC}$ & $61(25.3)$ & $96(32.2)$ & 1 & & 1 & \\
\hline & & AA & $59(24.5)$ & $48(16.1)$ & $1.934(1.175-3.183)$ & $0.009 *$ & $1.971(1.184-3.280)$ & $0.009 *$ \\
\hline & & $\mathrm{AC}$ & $121(50.2)$ & $154(51.7)$ & $1.237(0.829-1.844)$ & 0.298 & $1.256(0.835-1.888)$ & 0.274 \\
\hline \multirow[t]{3}{*}{ rs 17045754} & $\mathrm{C} / \mathrm{G}$ & GG & $164(66.4)$ & $204(68)$ & 1 & & 1 & \\
\hline & & $\mathrm{CC}$ & $12(4.8)$ & $7(2.3)$ & $2.132(0.821-5.539)$ & 0.120 & $2.295(0.867-6.077)$ & 0.095 \\
\hline & & $\mathrm{CG}$ & $71(28.8)$ & $89(29.7)$ & $0.992(0.683-1.442)$ & 0.968 & $0.914(0.622-1.342)$ & 0.646 \\
\hline \multirow[t]{3}{*}{ rs843720 } & $\mathrm{G} / \mathrm{T}$ & $\mathrm{TT}$ & $100(40.5)$ & $120(40)$ & 1 & & 1 & \\
\hline & & GG & $30(12.1)$ & $35(11.7)$ & $1.029(0.590-1.792)$ & 0.921 & $1.009(0.573-1.780)$ & 0.974 \\
\hline & & GT & $117(47.4)$ & $145(48.3)$ & $0.968(0.675-1.388)$ & 0.861 & $1.021(0.706-1.476)$ & 0.914 \\
\hline
\end{tabular}

a Minor alleles, OR: Odds ratio, CI: Confidence interval;

${ }^{\mathrm{b}}: p$ values were calculated using unconditional logistic regression after adjusting for sex and age;

$*: p \leq 0.05$.

Bonferroni correction was performed with $p \leq 0.00036(0.05 / 14)$ considered significant. 
Table 4: Logistic regression analysis of the association between the SNPs and colorectal cancer (adjusted for sex and age)

\begin{tabular}{|c|c|c|c|c|c|c|c|c|c|c|}
\hline \multirow{2}{*}{ SNP ID } & \multirow{2}{*}{$\begin{array}{c}\text { Minor } \\
\text { allele }\end{array}$} & \multicolumn{3}{|c|}{ Additive model } & \multicolumn{3}{|c|}{ Dominant model } & \multicolumn{3}{|c|}{ Recessive model } \\
\hline & & OR & $95 \%$ & $p$-value & OR & 950 & $p$-value & OR & $95 \% \mathrm{CI}$ & $p$-value \\
\hline rs67 & G & 304 & 1.01 & $0 *$ & 340 & 928 & 15 & 1.549 & 2.496 & 0.072 \\
\hline rs12621038 & $\mathrm{T}$ & 0.832 & $0.652-1.062$ & 0.140 & 0.805 & $0.558-1.162$ & 0.246 & 0.750 & $0.482-1.167$ & 0.202 \\
\hline rs 16 & $\mathrm{~A}$ & 0.920 & 0.706 & 38 & 0.891 & $0.630-1.260$ & 0.513 & 0.927 & $0.520-1.654$ & 0.798 \\
\hline rs843752 & G & 1.243 & $0.942-1.640$ & & 1.331 & $0.941-1.884$ & & 1.230 & $0.626-2.417$ & 0.548 \\
\hline rs10439478 & $\mathrm{C}$ & 0.885 & $0.692-1.133$ & 333 & 0.992 & $0.690-1.428$ & 0.967 & 0.667 & 1.057 & 0.085 \\
\hline rs843645 & G & 1.322 & $1.001-1.746$ & $0.049 *$ & 1.415 & $0.999-2.004$ & 51 & 1.421 & $0.723-2.795$ & 0.308 \\
\hline rs11125529 & A & 1.183 & $0.862-1.624$ & 0.299 & 1.097 & $0.761-1.581$ & 0.618 & 2.628 & $0.938-7.364$ & 0.066 \\
\hline rs12615793 & A & 1.136 & $0.833-1.549$ & & 1.043 & $0.727-1.496$ & & 2.422 & 6.365 & 0.073 \\
\hline rs843711 & $\mathrm{T}$ & 1.380 & $1.077-1.768$ & $0.011 *$ & 1.288 & 0.88 & 0.190 & 1.921 & $1.247-2.960$ & $0.003 *$ \\
\hline rs11896604 & G & 1.216 & $0.898-1.649$ & 0.207 & 1.151 & $0.801-1.655$ & 0.447 & 2.187 & $0.908-5.265$ & 0.081 \\
\hline rs843706 & A & 1.390 & $1.079-1.791$ & $0.011 *$ & 1.425 & $0.968-2.097$ & 0.073 & 1.703 & $1.101-2.633$ & $0.017^{*}$ \\
\hline rs 17045754 & $\mathrm{C}$ & 1.108 & $0.809-1.516$ & 0.524 & 1.009 & $0.699-1.458$ & 0.960 & 2.356 & $0.896-6.197$ & 0.082 \\
\hline rs843720 & $\mathrm{G}$ & 1.010 & $0.779-1.308$ & 0.943 & 1.018 & $0.717-1.447$ & 0.919 & 0.998 & $0.587-1.698$ & 0.995 \\
\hline
\end{tabular}

OR, odds ratio; CI: confidence interval.

$* p \leq 0.05$.

Bonferroni correction was performed with $p \leq 0.00036(0.05 / 14)$ considered significant.

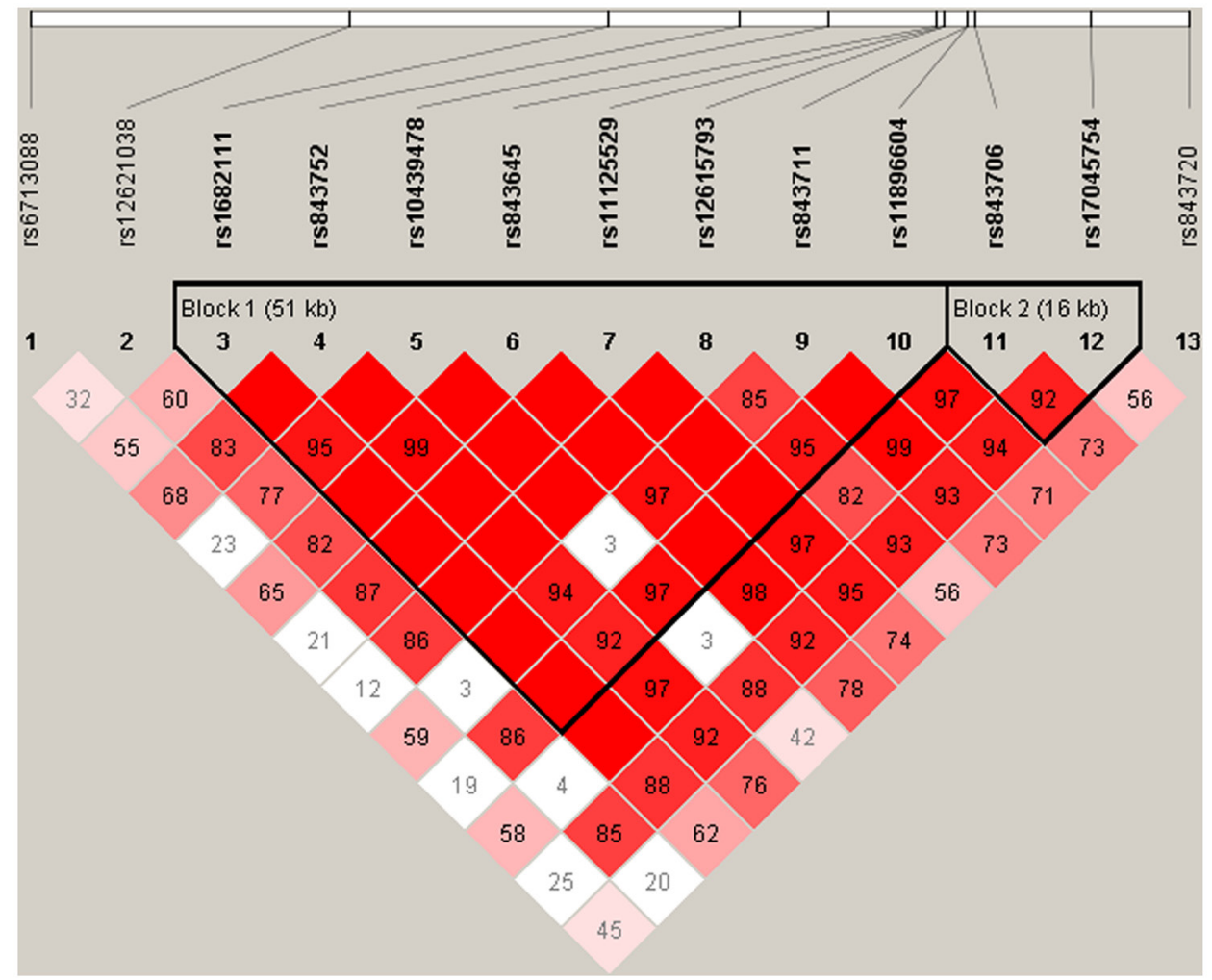

Figure 1: D' linkage map for the 13 SNPs in $\boldsymbol{A C Y P 2 . ~ A ~ s t a n d a r d ~ c o l o r ~ s c h e m e ~ w a s ~ u s e d ~ t o ~ d i s p l a y ~ l i n k a g e ~ d i s e q u i l i b r i u m ~ ( L D ) , ~}$ with bright red corresponding to strong $\mathrm{LD}\left(\mathrm{LOD}=2, D^{\prime}=1\right)$, white corresponding to no $\mathrm{LD}\left(\mathrm{LOD}<2, D^{\prime}<1\right)$, and pink $/$ red $(\mathrm{LOD}=2$, $\left.D^{\prime}<1\right)$ and blue (LOD $\left.<2, D^{\prime}=1\right)$ corresponding to intermediate LD. 
Table 5: $A C Y P 2$ haplotype frequencies and the association with the risk of colorectal cancer in cases and controls

\begin{tabular}{|c|c|c|c|c|c|c|c|c|c|c|}
\hline $\begin{array}{l}\text { Block } \\
\text { ID }\end{array}$ & SNPs & Haplotype & $\begin{array}{c}\text { Freq } \\
\text { (case) }\end{array}$ & $\begin{array}{c}\text { Freq } \\
\text { (control) }\end{array}$ & $\chi^{2}$ & $\begin{array}{c}\text { Pearson's } \\
p\end{array}$ & OR & $95 \%$ & $\mathrm{CI}$ & $p$-adj \\
\hline \multirow{5}{*}{1} & \multirow{5}{*}{$\begin{array}{l}\text { rs1682111, rs843752, } \\
\text { rs10439478, rs843645, } \\
\text { rs11125529, rs12615793, } \\
\text { rs843711, rs11896604 }\end{array}$} & TGAGCGTC & 0.279 & 0.231 & 3.279 & 0.070 & 1.325 & 1.001 & 1.754 & 0.050 \\
\hline & & ATATCGCC & 0.303 & 0.327 & 0.674 & 0.412 & 0.930 & 0.713 & 1.213 & 0.592 \\
\hline & & TTCTAATG & 0.201 & 0.172 & 1.506 & 0.220 & 1.184 & 0.862 & 1.626 & 0.298 \\
\hline & & TTCTCGCC & 0.182 & 0.227 & 3.289 & 0.070 & 0.719 & 0.529 & 0.978 & $0.035^{*}$ \\
\hline & & TTCTCACC & 0.008 & 0.015 & 1.092 & 0.297 & 0.542 & 0.160 & 1.837 & 0.326 \\
\hline \multirow{4}{*}{2} & \multirow{4}{*}{ rs843706, rs17045754 } & $\mathrm{AC}$ & 0.185 & 0.166 & 0.636 & 0.425 & 1.107 & 0.805 & 1.523 & 0.532 \\
\hline & & $\mathrm{AG}$ & 0.311 & 0.253 & 4.431 & 0.035 & 1.377 & 1.044 & 1.815 & $0.023 *$ \\
\hline & & CG & 0.504 & 0.576 & 5.469 & 0.019 & 0.732 & 0.568 & 0.944 & $0.016^{*}$ \\
\hline & & $\mathrm{TA}$ & 0.171 & 0.139 & 2.144 & 0.143 & 1.294 & 0.925 & 1.811 & 0.133 \\
\hline
\end{tabular}

OR: odd ratio; CI: confidence interval.

$p$-adj: $p$-values were adjusted for sex and age;

${ }^{*} p$ value $\leq 0.05$.

Bonferroni correction was performed, with $p \leq 0.00036(0.05 / 14)$.

Table 6: Primers used in this study

\begin{tabular}{llll}
\hline SNP_ID & 1st_PCRP & 2st_PCRP & UEP_SEQ \\
\hline rs6713088 & ACGTTGGATGACACACACAGACTCCTTCAC & ACGTTGGATGGTCACCAAAACACGTAATG & gaggcCAGAATGGTCCACTAGAGA \\
rs12621038 & ACGTTGGATGATTGTGCTAGGCACTTTAGG & ACGTTGGATGGGCATAAGTTTTATTGCCTC & ccATTGCCTCAGCTAGACT \\
rs1682111 & ACGTTGGATGGAATTGCTGGGTTATTTGGC & ACGTTGGATGGCCAGTGGGAATGCAAAATG & tgtcATGCAAAATGAAACAGACACTT \\
rs843752 & ACGTTGGATGTCCTCTTTTCAGAAACCTGC & ACGTTGGATGGAGACAACATAATGGAGGTC & cGAGTTTGGGTTTGAGGT \\
rs10439478 & ACGTTGGATGTAGCACAAGACCTACACTGG & ACGTTGGATGCTACACTCTCCAGAGGAATG & TTGCTGTTTTCCCAGAA \\
rs843645 & ACGTTGGATGACAGTGCCTTTAGCAAGGTG & ACGTTGGATGGAAATCTGAATACCACCTAC & TCATAGGCACTACTGTATC \\
rs11125529 & ACGTTGGATGCCGAAGAAAAGAAGATGAC & ACGTTGGATGGAGCTTAGTTGTTTACAGATG & AGAAAAGAAGATGACTAAAACAT \\
rs12615793 & ACGTTGGATGATCTTGGCCCTTGAAGAA & ACGTTGGATGTTTGAGCTTAGTTGTTTAC & AAATTGAGTGACAAATATAAACTAC \\
rs843711 & ACGTTGGATGTGCCTTGTGGGAATTAGAGC & ACGTTGGATGGACAAAGGACCTTACAACTC & gggaTCAGGGAACCAGTGCAAA \\
rs11896604 & ACGTTGGATGTGTCTCTGACCTAGCATGTA & ACGTTGGATGAAGTCAGAATAGTGCTTAC & GTTAAGCTTGCAAGGAG \\
rs843706 & ACGTTGGATGTGAATAACTTGGTCTTATC & ACGTTGGATGTGAAAGCCATAAATATTTTG & cACTTGGTCTTATCTGATGC \\
rs17045754 & ACGTTGGATGCTGTAAAAGTTCTGGCATGG & ACGTTGGATGGAAATCAGGGATATTAGTGC & caggTATTCAGCTTCCTAGAGTTA \\
rs843740 & ACGTTGGATGTCACAGACCCCCATAGTTAG & ACGTTGGATGTGAGGAAACTGAAGTTTAGC & ccctcTTGCTTCTTGGGGCCTAACA \\
rs843720 & ACGTTGGATGCTTCACAACACTCCTGTAAG & ACGTTGGATGAGTCAGAGCTAGACCTCTGG & ccccAATCTGTCTCAGGGTCTT \\
\hline
\end{tabular}

in regulating glucose and pyruvate metabolism in CRC patients. However, the mechanistic details have not yet been elucidated.

Telomeres have a critical role in maintaining genomic stability [22, 23]. Telomere lengths become shorter following each cell division. Therefore, telomere length could serve as a marker of cellular and biological age [24]. It is possible that shortened telomeres may predispose individuals to certain diseases. Genome-wide association studies have demonstrated that rs11125529 in the $A C Y P 2$ gene was associated with reduced telomere length $[12,25]$. Although we did not observe an association between rs11125529 and CRC risk in our study population, we did find that rs6713088, rs843645, rs843711, and rs843706 were associated with CRC risk. Furthermore, we found that the "TTCTCGCC" and "CG" haplotypes were protective against $\mathrm{CRC}$ while the " $\mathrm{AG}$ " haplotype may increase the risk of CRC. These results suggested that genetic variations in the $A C Y P 2$ gene may impact $\mathrm{CRC}$ risk by influencing telomere length.

The Bonferroni correction is commonly used to address false discovery rates resulting from multiple 
comparisons. We found that only the association between rs843711 and CRC was significant after Bonferroni correction, while rs843706, rs6713088, and rs843645 did not have a significant association with $\mathrm{CRC}$. This may be due to our strict SNP filtering criteria and small sample size. The Bonferroni correction adjusts the value of alpha based on the number of tests performed and is relatively conservative. In some cases, truly significant differences may be deemed non-significant as a result of type II errors [26].

Our study had several intrinsic limitations. For example, $\mathrm{CRC}$ is a heterogeneous disease, and alcohol and tobacco consumption are important risk factors for CRC. Because our study had a relatively small size, and it did not incorporate data regarding alcohol and tobacco consumption, we could not explore the interactions between genetic polymorphisms and environmental factors in CRC patients. Therefore, the relationship between $A C Y P 2$ polymorphisms and drinking and smoking status in CRC must be evaluated in future studies.

In summary, our results indicate that SNPs in the $A C Y P 2$ gene are associated with CRC in a Chinese Han population. These SNPs may serve as a prognostic biomarkers for $\mathrm{CRC}$ in the Chinese population. Future studies will focus on elucidating the function of $A C Y P 2$ in $\mathrm{CRC}$, which could be important for CRC prevention or early detection, and for improving patient prognosis.

\section{MATERIALS AND METHODS}

\section{Study participants}

All participants in our study were Han Chinese. A total of 247 patients and 300 controls were consecutively recruited between January 2011 and December 2014 at the LiaoNing Cancer Hospital and Institute, Affiliated Tumor Hospital of China Medical University, China. There were no gender, age, or disease stage restrictions for case recruitment. All cases were previously healthy individuals. The diagnosis of CRC was confirmed by histopathological examination. The control subjects had personal history of malignancy or chronic disease. We excluded patients who underwent radiotherapy or chemotherapy, as well as controls with chronic diseases. All participants in the current study were not related by blood.

\section{Clinical data and demographic information}

We used a standard epidemiological questionnaire and in-person interview to collect personal data including age, gender, residential region, education status, and family history of cancer. The case information was collected through consultation with the treating physicians or from medical chart reviews. All of the participants provided written informed consent. The Human Research Committee for Approval of Research Involving Human
Subjects, LiaoNing Cancer Hospital and Institute, Affiliated Tumor Hospital of China Medical University, approved the use of human blood samples in this study.

\section{SNP selection and genotyping}

Among the 14 SNPs we selected, rs11125529 was chosen based on previously published polymorphisms associated with telomere length [12]. The others were randomly selected. All of the SNPs had MAFs $>5 \%$ in the HapMap Chinese Han Beijing population. DNA was extracted from whole blood samples using the GoldMagMini Whole Blood Genomic DNA Purification Kit (GoldMag Co. Ltd. Xi'an City, China). Quantification of the extracted DNA was performed using a NanoDrop 2000 (Thermo Scientific, Waltham, Massachusetts, USA). The multiplexed SNP MassEXTENDED assay was designed using the Sequenom MassARRAY Assay Design 3.0 Software [27]. Genotyping was performed on a Sequenom MassARRAY RS1000 platform using the manufacturer's protocol. The PCR primers for each SNP are shown in Table 6. Data management and analysis was performed using the Sequenom Typer 4.0 Software [27, 28].

\section{Statistical analysis}

We used Microsoft Excel and the SPSS 18.0 statistical package (SPSS, Chicago, IL, USA) to perform statistical analyses. All $p$ values presented in this study were two sided, and $p=0.05$ was considered the cutoff for statistical significance. Differences in the characteristics of the case and control study populations were analyzed using chi-square tests for categorical variables and Welch's t tests for continuous variables. In all analyses, the lower frequency allele was considered to be the 'risk' allele. Control genotype frequencies for each SNP were tested for departure from HWE using Fisher's exact tests. Allele and genotype frequencies in the cases and controls were compared using chi-square tests [29]. Three models (additive, dominant, and recessive) were used to assess the association between each genotype and the risk of CRC. The effects of the polymorphisms on the risk of CRC were expressed as ORs with 95\% CIs, which were calculated using unconditional logistic regression analysis after adjusting for age and gender [30]. Finally, LD patterns and haplotypes were evaluated using the Haploview software package (version 4.2) [31]. Bonferroni correction was performed on all $p$ values, and the threshold for statistical significance was set at $p \leq 0.00036(0.05 / 14)$.

\section{ACKNOWLEDGMENTS}

We are grateful to Dr. MingDi Yin for performing the statistical analysis. We also thank Yu Liu for providing technical support and performing the SNP association analysis. 


\section{CONFLICTS OF INTEREST}

The authors declare that there are no conflicts of interest.

\section{GRANT SUPPORT}

This work was supported by grants from the $\mathrm{Wu}$ JiePing Medical Foundation (No. 320.6750 .16063 to Zhong-guo Zhang) and the Project of the Scientific Research Foundation of Liaoning Province (No. 2016003002 to Zhong-guo Zhang).

\section{REFERENCES}

1. Center MM, Jemal A, Ward E. International trends in colorectal cancer incidence rates. Cancer epidemiology, biomarkers \& prevention. 2009; 18:1688-1694.

2. Jemal A, Siegel R, Ward E, Hao Y, Xu J, Murray T, Thun MJ. Cancer statistics, 2008. CA Cancer J Clin. 2008; 58:71-96.

3. Lichtenstein P, Holm NV, Verkasalo PK, Iliadou A, Kaprio J, Koskenvuo M, Pukkala E, Skytthe A, Hemminki K. Environmental and heritable factors in the causation of cancer-analyses of cohorts of twins from Sweden, Denmark, and Finland. N Engl J Med. 2000; 45:167-168.

4. de Lange T, Shiue L, Myers RM, Cox DR, Naylor SL, Killery AM, Varmus HE. Structure and variability of human chromosome ends. Molecular and cellular biology. 1990; 10:518-527.

5. de Lange T. Shelterin: the protein complex that shapes and safeguards human telomeres. Genes \& development. 2005; 19:2100-2110.

6. Blasco MA. Telomeres and human disease: ageing, cancer and beyond. Nature reviews Genetics. 2005; 6:611-622.

7. Hug N, Lingner J. Telomere length homeostasis. Chromosoma. 2006; 115:413-425.

8. Huffman KE, Levene SD, Tesmer VM, Shay JW, Wright WE. Telomere shortening is proportional to the size of the G-rich telomeric 3'-overhang. The Journal of biological chemistry. 2000; 275:19719-19722.

9. Allsopp RC, Vaziri H, Patterson C, Goldstein S, Younglai EV, Futcher AB, Greider CW, Harley CB. Telomere length predicts replicative capacity of human fibroblasts. Proceedings of the National Academy of Sciences of the United States of America. 1992; 89:10114-10118.

10. Wu X, Amos CI, Zhu Y, Zhao H, Grossman BH, Shay JW, Luo S, Hong WK, Spitz MR. Telomere dysfunction: a potential cancer predisposition factor. Journal of the National Cancer Institute. 2003; 95:1211-1218.

11. Degl'Innocenti D, Marzocchini R, Rosati F, Cellini E, Raugei G, Ramponi G. Acylphosphatase expression during macrophage differentiation and activation of U-937 cell line. Biochimie. 1999; 81:1031-1035.
12. Codd V, Nelson CP, Albrecht E, Mangino M, Deelen J, Buxton JL, Hottenga JJ, Fischer K, Esko T, Surakka I. Identification of seven loci affecting mean telomere length and their association with disease. Nature genetics. 2013; 45:422-427.

13. Yu WS, Jeong SJ, Kim JH, Lee HJ, Song HS, Kim MS, Ko E, Lee HJ, Khil JH, Jang HJ, Kim YC, Bae H, Chen $\mathrm{CY}$, Kim SH. The genome-wide expression profile of 1,2,3,4,6-penta-O-galloyl-beta-D-glucose-treated MDA-MB-231 breast cancer cells: molecular target on cancer metabolism. Molecules and cells. 2011; 32:123-132.

14. Pooley KA, Bojesen SE, Weischer M, Nielsen SF, Thompson D, Amin Al Olama A, Michailidou K, Tyrer JP, Benlloch S, Brown J, Audley T, Luben R, Khaw K-T, et al. A genome-wide association scan (GWAS) for mean telomere length within the COGS project: identified loci show little association with hormone-related cancer risk. Human Molecular Genetics. 2013.

15. Yu WS, Jeong S-J, Kim J-H, Lee H-J, Song HS, Kim M-S, Ko E, Lee H-J, Khil J-H, Jang H-J. The genomewide expression profile of 1, 2, 3, 4, 6-penta-O-galloyl$\beta$-D-glucose-treated MDA-MB-231 breast cancer cells: molecular target on cancer metabolism. Molecules and cells. 2011; 32:123-132.

16. Won HH, Lee J, Park JO, Park YS, Lim HY, Kang WK, Kim JW, Lee SY, Park SH. Polymorphic markers associated with severe oxaliplatin-induced, chronic peripheral neuropathy in colon cancer patients. Cancer. 2012; 118:2828-2836.

17. Pradelli L, Villa E, Zunino B, Marchetti S, Ricci J. Glucose metabolism is inhibited by caspases upon the induction of apoptosis. Cell death \& disease. 2014; 5:e1406.

18. Conde VR, Oliveira PF, Nunes AR, Rocha CS, Ramalhosa E, Pereira JA, Alves MG, Silva BM. The progression from a lower to a higher invasive stage of bladder cancer is associated with severe alterations in glucose and pyruvate metabolism. Experimental cell research. 2015; 335:91-98.

19. Vaz CV, Alves MG, Marques R, Moreira PI, Oliveira PF, Maia CJ, Socorro S. Androgen-responsive and nonresponsive prostate cancer cells present a distinct glycolytic metabolism profile. The international journal of biochemistry \& cell biology. 2012; 44:2077-2084.

20. Israelsen WJ, Vander Heiden MG. (2015). Pyruvate kinase: function, regulation and role in cancer. Seminars in cell \& developmental biology: Elsevier), pp. 43-51.

21. Szlosarek PW, Lee S, Pollard PJ. Rewiring mitochondrial pyruvate metabolism: switching off the light in cancer cells? Molecular cell. 2014; 56:343-344.

22. Blackburn EH. Telomere states and cell fates. Nature. 2000; 408:53-56.

23. Wong JM, Collins K. Telomere maintenance and disease. The Lancet. 2003; 362:983-988.

24. Harris SE, Martin-Ruiz C, von Zglinicki T, Starr JM, Deary IJ. Telomere length and aging biomarkers in 70-year-olds: 
the Lothian Birth Cohort 1936. Neurobiology of aging. 2012; 33:1486. e1483-1486. e1488.

25. Pooley KA, Bojesen SE, Weischer M, Nielsen SF, Thompson D, Al Olama AA, Michailidou K, Tyrer JP, Benlloch S, Brown J. A genome-wide association scan (GWAS) for mean telomere length within the COGS project: identified loci show little association with hormone-related cancer risk. Human molecular genetics. 2013:ddt355.

26. Perneger TV. What's wrong with Bonferroni adjustments. Bmj. 1998; 316:1236-1238.

27. Gabriel S, Ziaugra L, Tabbaa D. SNP genotyping using the Sequenom MassARRAY iPLEX platform. Current protocols in human genetics. 2009:2.12. 11-12.12. 16.
28. Thomas RK, Baker AC, DeBiasi RM, Winckler W, LaFramboise T, Lin WM, Wang M, Feng W, Zander T, MacConaill LE. High-throughput oncogene mutation profiling in human cancer. Nature genetics. 2007; 39:347-351.

29. Adamec C. Example of the use of the nonparametric test. Test X2 for comparison of 2 independent examples. Ceskoslovenské zdravotnictví. 1964; 12:613.

30. Bland JM, Altman DG. Statistics notes. The odds ratio. BMJ (Clinical research ed). 2000; 320:1468.

31. Yong Y, Lin H. SHEsis, a powerful software platform for analyses of linkage disequilibrium, haplotype construction, and genetic association at polymorphism loci. Cell research. 2005; 15:97-98. 